\title{
Correction to: Mitochondrial Modulation by Dichloroacetate Reduces Toxicity of Aberrant Glial Cells and Gliosis in the SOD1G93A Rat Model of Amyotrophic Lateral Sclerosis
}

\author{
Laura Martínez-Palma ${ }^{1,2} \cdot$ Ernesto Miquel $^{1,2} \cdot$ Valentina Lagos-Rodríguez $^{1,2} \cdot$ Luis Barbeito $^{3} \cdot$ Adriana Cassina $^{2,4}$. \\ Patricia Cassina ${ }^{1,2}$
}

Published online: 20 May 2020

(C) The American Society for Experimental NeuroTherapeutics, Inc. 2020

\section{Neurotherapeutics 2019 Jan;16(1):203-215 \\ https://doi.org/10.1007/s13311-018-0659-7}

The authors would like to correct the following errors:

In Methods section, subsection Respirometry studies:

The statement in parentheses "(oligo-inhibited flux-AAinhibited flux)" should be deleted

The calculation of the RCR is wrong. The text "mitochondrial oligo-inhibited OCR / maximal mitochondrial respiratory capacity OCR" should be changed to "maximal mitochondrial respiratory capacity OCR / mitochondrial oligo-resistant respiration rate $(\mathrm{H}+\mathrm{leak})$ ".

The online version of the original article can be found at https://doi.org/ 10.1007/s13311-018-0659-7

Laura Martínez-Palma lmartinezpalma07@gmail.com

Patricia Cassina pcassina@fmed.edu.uy

1 Departamento de Histología y Embriología, Facultad de Medicina, Universidad de la República, Montevideo, Uruguay

2 Centro de Investigaciones Biomédicas (CEINBIO), Facultad de Medicina, Universidad de la República, Montevideo, Uruguay

3 Institut Pasteur de Montevideo, Montevideo, Uruguay

4 Departamento de Bioquímica, Facultad de Medicina, Universidad de la República, Montevideo, Uruguay
In Results section

In Fig.2a, bottom panel: the equation in the square "RCR = MRR / ATPP" is incorrect; it should be changed to "RCR = MRR / H+ leak".

Table 2 is corrected below.

The interpretation of the results and conclusions in the paper are not changed

Table 2 Effects of DCA on respiratory parameters

\begin{tabular}{|c|c|c|c|c|c|}
\hline \multirow[t]{2}{*}{ Parameter } & \multicolumn{2}{|c|}{ Value \pm SEM $(\mathrm{pmol} / \mathrm{ml} \cdot \mathrm{s})$} & \multirow[t]{2}{*}{$P$} & \multirow[t]{2}{*}{$T$} & \multirow[t]{2}{*}{ significance leve } \\
\hline & Ctrl & DCA & & & \\
\hline Basal & $59.4 \pm 8.42$ & $73.1 \pm 0.63$ & .1777 & 1.090 & $n s$ \\
\hline $\mathrm{H}+$ leak & $25.9 \pm 5.37$ & $28.4 \pm 0.49$ & .3207 & 0.516 & $n s$ \\
\hline ATPP & $38.5 \pm 6.92$ & $44.7 \pm 0.67$ & .0882 & 1.761 & $n s$ \\
\hline MRR & $47.1 \pm 9.51$ & $96.1 \pm 4.40$ & .0194 & 3.522 & $*$ \\
\hline SRC & $26.7 \pm 14.05$ & $41.7 \pm 9.42$ & .0373 & 2.686 & $*$ \\
\hline RCR & $2.0 \pm 0.34$ & $2.74 \pm 0.31$ & .0018 & 8.311 & $* *$ \\
\hline
\end{tabular}

Basal $=$ basal OCR, $\mathrm{H}+$ leak $=$ proton leak $($ mitochondrial oligo-resistant respiration OCR), ATPP = ATP production-linked OCR (oligomycin sensitive mitochondrial OCR) MRR = maximum respiratory rate (maximum FCCP induced mitochondrial OCR), $\mathrm{SRC}=$ spare respiratory capacity (MRC minus basal mitochondrial OCR). $n=4, d f=3, n s=$ nonsignificant $* p<0.05, * * p<0.01$

Publisher's Note Springer Nature remains neutral with regard to jurisdictional claims in published maps and institutional affiliations. 